\title{
A scoping review of insomnia treatments for people living with HIV
}

\author{
Review \\ Joyal Miranda ${ }^{1}$, Souraya Sidani ${ }^{1}$, José Côté ${ }^{2}$, Suzanne Fredericks ${ }^{1}$ \\ ${ }^{1}$ Daphne Cockwell School of Nursing, Ryerson University, Ryerson University, Toronto, Ontario, Canada; ${ }^{2}$ Faculty of \\ Nursing, Université de Montréal and Center de Recherche, University Hospital of Montreal (CRCHUM), Quebec, \\ Canada
}

Corresponding author: J. Miranda (joyal.miranda@ryerson.ca)

\section{ABSTRACT}

To date, little is known in terms of viable treatments for insomnia in people living with HIV. The primary aim of this scoping review is to identify non-pharmacological treatments for insomnia in people living with HIV. A framework by Arksey and O'Malley was used to guide the conduct of this scoping review. Seven studies were identified. Three of the studies used cognitive-behavioral type of treatments versus physical or alternative types of treatment. The most effective treatments with the largest effect sizes ( $E S=1.11$ to 1.91) were found to be cognitive-behavioral treatments for the sleep outcomes of sleep quantity and sleep quality. This review found that cognitive behavioral interventions were found to be the most effective treatments for insomnia for people living with HIV. Further research would benefit from larger sample size studies in addition to focusing on the determinants of insomnia in people living with HIV in order to further provide a treatment that is focused on the needs of people living with HIV.

\section{KEYWORDS}

People living with HIV, sleep disturbances, insomnia, cognitive-behavioral treatment, HIV/AIDS

\section{INTRODUCTION}

Sleep is a vital biological process to the everyday lives of individuals supporting optimal health, yet it is something that individuals struggle with daily. Sleep plays an important role in one's psychological state, cognitive performance, as well as functioning of immune, hormonal, and cardiovascular systems (Medic et al., 2017; Zielinski et al., 2016). It affects up to one-third of the general population, with $10-35 \%$ estimated to suffer from sleep disturbances (Low et al. 2014; Wu et al., 2015; Jabbari et al., 2015). Poor sleeping habits, lifestyle choices, psychological issues, environmental factors, medical conditions and medications are factors contributing to sleep disturbances (Medic et al., 2017). Insomnia is a sleep disturbance, characterized by difficulty initiating or maintaining sleep for at least three nights per week for three months or longer (American Psychiatric Association, 2013). Those diagnosed and living with insomnia may experience a low quality of life, low daytime

functioning, impaired cognitive functioning, and reduced work productivity, potentially increasing health services utilization and mortality rates (Bhaskar, Hemavathy, Prasad, 2016; Straten, Zweerde, Kleiboer, Cuijpers, Morin, Lancee, 2017). Furthermore, chronic insomnia may result in additional significant health problems among individuals who may already be dealing with chronic illnesses.

Currently, there is a significant number of studies including meta-analysis that have investigated various treatments for insomnia within the general population, as well as specific sub-populations with psychiatric and medical conditions such as depression, anxiety, cardiovascular disease and cancer (Chung et al., 2018; van Stratten et al., 2018; Gong et al., 2016; Wu, Appleman, Salazar, Ong, 2015; Geiger-Brown, Rogers, Liu, Ludeman, Downton, Diaz-

61 
Abad, 2015; Miranda, Sidani, Fredericks, Fox, 2017;Johnson, Rash, Campbell, Savard, Gehrman, Perlis, Carlson, Garland, 2016).

A common insomnia treatment that has been supported empirically is that of Cognitive Behavioral Treatment Insomnia (CBT-I) (Morin, Beaulieu_Bonneau \& Cheung, 2019). CBT-I is highly recommended because it involves psychological approaches to the management of insomnia; these approaches are likely to produce sustained benefits without incurring the risk for tolerance or adverse side effects that are often associated with pharmacological approaches (Trauer, Qian, Doyle, Rajaratnam, Cujnnignton, 2015). CBT-I is grounded in the theories of behavior change, psychology and sleep. With a strong empirical support, CBT-I is considered as the first-line treatment for insomnia (Quaseem, Forciea et al., 2016).

Even though there is strong empirical support for the effectiveness of CBT-I in various populations, few studies have focused on effects of treatment for People Living with HIV (PLWH). Several studies have systematically examined the experience of insomnia in PLWH as well as describing insomnia and factors contributing to insomnia in this population. (Taibi, 2013; Rogers et al., 2018; Jean-Louis et al., 2012; Jabbari et al., 2012, Wu et al., 2015). PLWH are a subpopulation reported to suffer from insomnia more so than the general population (Low et al. 2014; Wu et al., 2015; Jabbari et al., 2015). It is estimated that 29$97 \%$ of PLWH experiences sleep disturbances such as insomnia (Low et al., 2014). This estimate is well above that of the general population (Low et al. 2014; Wu et al., 2015; Jabbari et al., 2015). For instance, Webel et al. (2013), Jean-Lous et al. (2012), and Wu et al. (2015) reported insomnia as being one of the earliest and most distressing complaints of PLWH and as a frequently occurring symptom across all stages of the HIV disease. Some studies examined possible clinical factors for the high prevalence of insomnia within this population. Clinical factors included the type of HIV medication, time since diagnosis, viral load, CD4+ cell count and presence of other diseases (Low et al., 2014; Jean-Louis et al., 2012). These clinical factors contribute to insomnia above and beyond the factors of age, sex, physical health and mental health status that are also prevalent in this sub-population and others as well as the general population (Garland, Rowe, Repa, Fowler, Rhou \&
Grandner, 2018; Morin, LeBlanc, Belanger, Ivers, Chantal, Merette \& Savard, 2011).

The impact of insomnia on PLWH may be more severe than that of the general population or other sub-populations given the additional clinical factors being considered such as disease exacerbation. Not only does insomnia affect a greater proportion of PLWH than the general population, but it also has significant effects on the individual, that also surpass those experienced by the general population. Untreated insomnia in PLWH may lead to poorer disease outcomes; poor medication adherence which further complicates clinical management of the disease as it may cause loss of virologic control. Individuals may also develop drug resistant strains of the disease leading to treatment failure (Low et al., 2014; Webel etal., 2013; Rogers et al., 2018; JeanLouis et al., 2012; Wu et al., 2015; Jabbari et al., 2015). Lastly, insomnia also affects the individual's immune status which is already compromised by the disease itself (Low et al., 2014).

\section{AIM}

The inter-relationship among sleep, the immune system and HIV infection underscores the importance of examining insomnia in PLWH and treatments for the management of insomnia in PLWH. To date, little is known in terms of viable treatments for insomnia in PLWH; therefore, the primary aim of this scoping review is to identify non-pharmacological treatments for insomnia in PLWH.

\section{METHODS}

A scoping review was undertaken in order to address the broad topic of insomnia interventions for PLWH regardless of the type of research designs used. In particular, Arksey and O'Malley's (2005) framework was used to guide the scoping review. A key strength of the framework is that it provides a rigorous and transparent method for mapping areas of research, making it possible to identify and describe interventions found effective as well as identify the gaps in the evidence base (Arksey and O'Malley (2005). The framework encompasses five steps.

\section{Step 1: Identifying the Research Question}

The main research question for this scoping review was: What non-pharmacological treatments have 
been used to address or manage insomnia in PLWH? The secondary aims of the study are to identify: 1) how the term insomnia is defined within the studies; 2) data collected relevant to HIV status and insomnia; 3) the types of treatments used to manage insomnia; and 4) the outcomes of the treatments, in particular those related to insomnia.

\section{Step 2: Identifying Relevant Studies}

The search was conducted with no restrictions on time of publication, in order to capture a broader understanding of the types of studies conducted in the area of insomnia treatment and PLWH. The search was done in the following databases: Medline, PsychInfo, CINAHL and PubMed. The search terms or key words included: insomnia, sleep disruption, HIV, People living with HIV, treatment, therapy and intervention. In each database, the search was limited to peer-review and excluded books. Search modes and expanders included finding all search terms and matching terms to subject heading. Once all database searches were completed, there were a total number of 899 hits. Each individual database search was uploaded into a document file. The individual files were then merged into one file in order to detect duplicates. Of the original 899 hits, 398 were identified as duplicates and removed, leaving 501 articles to be reviewed for eligibility into the scoping review. Prior to the screening of the titles and abstracts, eligibility criteria for the articles were discussed and agreed upon by the full research team. The principal investigator did the initial screening of titles and abstracts. In order to verify reliability of screening, a random sample of articles from the literature search were chosen in which an additional researcher from the team also screened the title and abstracts for initial eligibility. There was 95\% agreement in terms of the screening of title and abstracts for eligibility in addition to the coding of the rationales for excluding the articles. From the title and abstract screening, a total of 7 studies were deemed eligible. A full-text review of the 7 studies was undertaken by the full research team in which $100 \%$ agreement was obtained to include the 7 studies in the scoping review. The reference lists of the selected studies were also reviewed by the principal investigator in order to locate additional relevant studies that may have been missed in the database searches. This additional search did not retrieve additional studies.

\section{Step 3: Study Selection}

Studies were selected if: the target population was comprised of people living with HIV; a treatment was given to manage insomnia; and treatments were of a non-pharmacological nature. In reviewing the title and abstract of the 501 articles, 494 were deemed as non-eligible because: 1) the population under study were PLWH but there was no focus on insomnia $(n=279) ; 2)$ studies examined and mainly described the experience of insomnia and PLWH and did not provide any treatment ( $n=126)$; 3) main focus was the pharmacological treatment of insomnia for PLWH $(n=5)$; 4) studies addressed insomnia and/or treatment but did not include PLWH $(n=22)$ and 5) studies in which the terms (insomnia, insomnia treatments or PLWH) were mentioned in the article but were not the focus of the study $(n=62)$. A total of seven studies were included in the scoping review. See Figure 1 for flow of studies into the scoping review.

\section{Step 4: Charting of Data}

The data extraction chart was initially designed by the principal investigator, reviewed and approved by the co-investigators. The data extraction items included: study authors, publication date and title; study aim/objective; study population (including number of participants and inclusion/exclusion criteria); whether insomnia was explicitly defined (definition provided); measures of insomnia (including sleep parameters such as sleep duration); description of the treatment provided; and results related to the effects of the treatment on insomnia.

Step 5: Collating, Summarizing and Reporting the Results

The results obtained from the data extracted in step 4 were synthesized where possible and are discussed in the results section.

\section{RESULTS}

\section{Characteristics of the Selected Studies}

Table 1 identifies the characteristics of the studies included in the scoping review. Majority (86\%) of the studies were from the United States of America $(n=6)$; the majority (58\% to $81 \%$ ) of participants were men. On average, the mean age of the participants ranged 
from 40 to 48 years. All studies $(n=7)$ used some variation of an experimental design, with the majority (71\%) using a randomized controlled design with a notreatment or instructed to maintain usual daily routine $(n=5)$ versus treatment $(n=2)$ as the control group. Three studies (Buchannan et al., 2018; Hudson et al., 2008 and Webel et al., 2013) provided treatments of a cognitive behavioral nature, whereas the remaining four studies (Phillips \& Skelton, 2001; McDermott et al., 2017; Sandoval et al., 2016 and Dreher, 2003) provided alternative treatments such as acupuncture, exercise, splinting for lower extremities and decreasing caffeine consumption. Three of the seven studies Dreher, 2003; Buchanan et al., 2018 and Philip \& Skelton, 2001) screened for insomnia using validated measures such as the Pittsburgh Sleep Quality Index (PSQI) and DSM-IV, while four studies (Hudson et al., 2008; Buchanan et al., 2018; McDermott et al., 2017 and Sandoval et al., 2016) included exclusion criteria that ranged from cognitive impairment, mental illness and sleep apnea. Instruments used to measure insomnia varied dependent on how insomnia was defined and operationalized. For instance, majority of the studies $(n=7)$ insomnia was represented as sleep quality and measured by PSQI (Sandoval et al., 2016; McDermott et al., 2017; Philips \& Skelton, 2001 and Dreher, 2003). Whereas in other studies $(n=4)$, insomnia was measured as sleep activity/quantity and assessed by wrist actigraphy and/or sleep diaries (Philip \& Skelton, 2001; Husdon et al., 2008; Buchanan et al., 208 and Webel et al., 2013). Each of the measures used are validated measures that are consistently used in insomnia research.

\section{Defining Insomnia}

Three of the seven studies included a general definition of insomnia above and beyond the description of insomnia as measured in the study (Buchanan et al., 2018; Hudson et al., 2008; Phillips \& Skelton, 2001). Insomnia was defined as difficulty falling asleep, awakening during the night, early morning awakenings and reduced sleep time. Of these three studies only one (Phillips \& Skelton, 2001) included the occurrence of insomnia as 3 or more nights per week. None of the studies included the duration of insomnia experience. The remaining studies (Dreher (2003); McDermott et al., 2017; Sandoval et al., 2016 and Weber et al., 2013) conceptualized insomnia as per the instruments being used to measure insomnia, which is discussed later in the paper.

\section{Data Collected Relevant to HIV/AIDS Status and Insomnia}

All participants in the selected studies $(n=7)$ were living with HIV. All studies $(n=7)$ collected data in relation to HIV/AIDS; the data reflected: years since HIV/AIDS diagnosis, route of infection, CD4+ cell count, HIV viral load, undetectable viral load and whether or not on antiretroviral medications was prescribed / taken and duration. Dreher (2003) was the only study to examine potential confounding variables (HIV related health status) and sleep quality and well-being. Results indicated that in the experimental group, those decreasing their caffeine intake experienced a significant improvement in their HIV-related health status (Physical Health Status Scores, $\mathrm{t}=3.323, \mathrm{p}=.002$; Mental Health Status scores, $\mathrm{t}=-3.646 ; \mathrm{p}=.001$ ) as opposed to the control group that showed no significant improvement. Dreher (2003) also examined any drug changes and their effect on sleep quality and well-being and found no change.

\section{Types of Treatment for Insomnia and Components of Treatment}

A variety of treatments for insomnia in PLWH were examined in the selected studies. Three studies (Hudson et al., 2008; Buchanan et al., 2018 and Webel et al., 2013) offered treatments that were of a cognitive behavioural nature. Of these, Buchanan et al. (2018) was the only study to include all components of Cognitive Behavioral Therapy (CBT), that is, sleep education, sleep restriction, stimulus control and circadian mechanisms. The treatment was four-weeks long and included both face-to-face and telephone sessions. Both Hudson et al. (2008) and Webel et al. (2013) focused only on the component of sleep hygiene as part of the insomnia treatment, but did so in an unconventional way; specifically, they evaluated a 10 -week sleep hygiene and behavioral modification strategy that focused on redesigning the individuals' system of interpersonal environment and daily routines linked to the health behavior. In other words, the focus was on changing the environment more so than the focusing on personal efforts to change individual behaviours. Hudson et al. (2008) on the other hand focused on a 1-week tailored sleep hygiene treatment in which 
participants were first educated on the various sleep hygiene behaviors and then, they were able to choose two from the potential six sleep hygiene behaviors for 1-week.

Four of the seven studies included physical or alternative treatments for insomnia (Phillips et al., 2001 and Sandavol et al., 2016 and McDermott et al., 2017; Dreher, 2003). For example, a 16-week aerobic exercise program, acupuncture with two-sessions per week for five weeks, use of splinting for lower extremities for six-weeks and decreasing caffeine consumption for 30-days were the treatments provided to individuals in order to decrease their insomnia.

\section{Outcomes}

Insomnia was measured in different ways in the studies targeting PLWH. Sleep quantity was measured by the use of sleep diaries where sleep quantity was captured through total sleep time, sleep onset latency, wake after sleep onset and sleep efficiency (Buchanan et al., 2018 and Hudson et al., 2008). Wrist actigraphy was also used to capture sleep quantity (Webel et al., 2013 and Husdon et al., 2008). When not reported in the article, the effect sizes were calculated as the standard difference in the means on the indicator for insomnia between treatment groups. The effect sizes were calculated in order to identify treatments that are most effective in managing insomnia in this population. The treatment with the largest effect size for sleep quantity (1.111.91) was the brief behavioral treatment based on the principles of CBT (Buchanan et al., 2018). The SystemCHANGE treatment based on the principles of sleep hygiene, behavioral modifications and the sociological model resulted in medium effects sizes $(0.40-0.50)$ (Webel et al., 2013). Lastly, the tailored sleep promotion intervention based on principles of sleep hygiene demonstrated small effect sizes (0.010.25 ) with the use of sleep diaries and medium effect sizes ( 0.02 to 0.5 ) on the circadian rhythm parameters (Hudson et al., 2008).

The treatment with the largest effect size noted for sleep quality was again the brief behavioral treatment (1.11- 1.91) (Buchanan et al., 2018), followed by the use of caffeine reduction (0.75) (Dreher, 2003). Both the SystemCHANGE and exercise as a treatment for insomnia also demonstrated medium effects on sleep quality ranging from $0.40-0.37$. (Webel et al., 2013 \&
McDermott et al., 2017). The remaining treatments for lower extremity splinting and tailored sleep promotion demonstrated small effect sizes ranging from 0.01 to 0.02 for sleep quality (Hudson et al., 2008 \& Sandoval et al., 2016).

Effect sizes for sleep impairment were found to be large (1.11 to 1.91) in the brief behavioral treatment (Buchanana et al., 2018) and small (0.1) in the SystemCHANGE treatment (Webel et al., 2013). Effect sizes for sleep disturbance were also noted to be medium (0.64) with the tailored sleep promotion treatment (Hudson et al., 2008), yet small (0.05) with the SystemChange treatment (Webel et al., 2013). Lastly, perceived severity of insomnia was only measured by Buchanan et al. (2018) yet was demonstrated to have a large effect size with the brief behavioral treatment (1.11-1.91).

\section{DISCUSSION}

Evaluation of pharmacological to nonpharmacological treatments for insomnia is widespread the general population but rather limited in PLWH. This review identified seven studies that evaluated non-pharmacological treatments for insomnia within PLWH. Results identified the treatment that tended to have the largest effect sizes on sleep quality and sleep quantity (ranging from 1.11 to 1.91) as the brief behavioral treatment (Buchanan et al., 2018) The brief behavioral treatment was comprised of 4 weekly sessions: two in person and two by telephone. Components of the treatment consisted of sleep hygiene, sleep restriction (limiting the time in bed when not asleep), stimulus control (avoiding conditioned sleep wakefulness in the sleep setting) as well as circadian mechanisms (keeping stable sleep schedule). These components are based on the four principles to promote sleep that is; reduced time in bed, keeping the same wakeup time regardless of sleep quality, going to bed only when sleepy and getting out of bed at night when not sleeping (Troxel et al., 2012). CBT-I has long been recommended as the first line of treatment for insomnia. The short-term effects of CBT-I are comparable to those of pharmacotherapy; CBT-I has also demonstrated efficacy in the long term (Taylor \& Pruiksma, 2014).

Results of several systematic review demonstrates that the components of CBT-I with the strongest empirical support in the general population, are 
stimulus control treatment (SCT), sleep restriction treatment (SRT), relaxation and cognitive therapy (Morgenthaler et al., 2006; Morin et al., 2006; Van Straten et al., 2018). As well, a study by Epstein et al (2012), has demonstrated that SCT, SRT and multicomponent interventions $(\mathrm{MCl})$ are equally efficacious and produce sustainable treatment gains on sleep outcomes. In comparison, treatments that solely focused on sleep hygiene were considered inadequate having overall smaller effects than CBT-I (Taylor \& Pruiksma, 2014). This was comparable to the findings reported by Hudson et al., (2008); the tailored sleep promotion treatment comprised of a 30-40-minute session focused on an educational and behavioral set of sleep promoting behaviors based on the principles of sleep hygiene only. Participants were asked to choose two of the six sleep hygiene principles to follow for a one-week period. Results indicated that the intervention had small effect sizes for sleep quality (0.01) and a small to medium effect size for sleep quantity (0.02-0.5). Dreher (2003) included a single component of sleep hygiene education focused on decreasing caffeine consumption over a 30-day period, while McDermott et al (2017) provided a 16-week exercise program. Each of these foci on the lifestyle and environmental factors related to sleep. The reduction of caffeine for 30 days resulted in an effect size of 0.75 for sleep quality, whereas the introduction of a 16-week exercise program resulted in an effect size of 0.37 for sleep quality.

The number of sessions in a CBT-I have also been debated in the literature. Previously CBT-I typically required 8-10 weekly sessions, but recently interventions of shorter duration that focus on behavioral principles have shown to be just as efficacious (Buchanan et L., 2018 \& Sidani et al., 2019). Thus, the ability to provide a shorter duration intervention without compromising the efficacy of the intervention has two implications; 1) it may be cost effective than longer session treatments and 2) may decrease the burden put on individuals to attend numerous sessions.

The selected studies had weaknesses. All seven studies had small sample sizes; with three studies classified as pilot studies examining the feasibility and preliminary efficacy of the treatments. Pilot studies do have value in that they help to address process measures, consent rate, rates of treatment flexibility, compliance, method of randomization, and outcome measures (Sim, 2019). Pilot studies also provide value in that they may highlight processes that did not work and provide alternative suggestions for future research. Buchanan et al., (2018) identified in their pilot study that a randomized wait-list design was an unsuccessful approach contributing to a high drop out rate. The author proposed future research should include an active control to retain participants. Whereas, Sandovoal et al. (2016) also noted high attrition rates, although did not provide a possible solution to address the issue in future research studies. Identification of issues and possible solutions support future research studies with the best research process based on previous pilot studies. In addition, many may also report on the preliminary efficacy of the treatment. Yet, effect sizes may be imprecise in small sample studies and therefore do not allow for a robust decision on a main trial (Sim, 2019). In order to further progress, replication studies with larger sample sizes are necessary.

Varied definitions of insomnia both conceptually and operationally is also problematic. As was seen in this scoping review, three of the seven studies included a general definition of insomnia where there was variability. A more liberal definition of insomnia tended to be used more often. The liberal definition focuses on the presence of nocturnal insomnia symptoms (e.g., difficulties with sleep initiations or maintenance, nonrestorative sleep). A more conservative definition requires additional features such as associated daytime impairment of which none of the studies included in their general definition of insomnia. As well the frequency of occurrence and duration of insomnia was mostly lacking in the general definitions. The use of varied definitions may lead to drastically different findings and conclusions regarding the general prevalence of insomnia as well as overall study results (Ohayon, 2002). Therefore it is important to include a standardized definition that is based on the most recent DSM-5. The DSM-5 identifies insomnia as a predominant complaint of dissatisfaction with sleep quantity or quality, associated with one (or more) of the following symptoms: difficulty initiating sleep, difficulty maintaining sleep, characterized by frequent awakenings or problems returning to sleep after awakenings, early-morning awakening with inability to return to sleep, sleep difficulty occurs for at least 3 nights per week and is present for at least 3 months (American Psychiatric Association, 2013). 
Additionally, there is a plethora of information relating to the experiences, potential determinants and correlates of insomnia and PLWH, although research examining the effects of such variables on insomnia in PLWH is minimal as well as inconsistent (Reid \& Dwyer, 2005). HIV-related clinical variables such as CD4+ cell counts and viral load may be associated with insomnia, but some studies have not supported this association (Taibi, 2012). A study by Jean-Louis et al. (2012), noted the duration of HIV infection, CD4+ cell count, viral load, lipoatrophy and HIV therapy type were not significantly associated with insomnia symptoms. As well, a systematic review based on 29 studies identified that the role of immune dysregulation, virus progression and adverse drug effects contributing to insomnia as unclear, whereas the antiretroviral treatment efavirenze was found to be a significant risk factor to insomnia (Reid \& Dwyer, 2005). Although research has also shown that sleep disturbing effects of efavirenze appears to decrease with time (e.g., 35\% report sleep disturbance after 4-weeks of therapy, $7 \%$ report sleep problems after 24-weeks of therapy and none report sleep problems at 48-weeks of therapy) (Low et al. 2014). The number of published studies that systematically evaluate the risk of insomnia associated with antiretroviral drugs is limited. Of the studies examining insomnia and PLWH, for many the effect of antiretroviral therapy was either not considered or participants receiving antiretroviral treatment were excluded (Reid \& Dwyer, 2005). Buchanan et al (2018) was the only study in this review to exclude individuals who were currently taking efavirenz for the sole purpose that it was known to cause sleep disturbance.

In this scoping review, all studies collected data relevant to individual's HIV/AIDS status such as: years since HIV/AIDS diagnosis, CD4+ cell count, HIV viral load, undetectable viral load and whether or not on antiretroviral medications and duration. The data collected were used for descriptive purposes. Dreher (2003) was the only study that examined potential confounding variables (HIV related health status) and sleep quality, although no significant effects were found. Behavioral and cognitive factors such as excessive worrying during nighttime awakenings and extended time in bed have also been found to contribute to insomnia in the general population but research with PLWH is typically lacking in this area as well (Taibi, 2012). Due to the limited research and inconsistencies in physiological, behavioral and cognitive factors that may play a role in PLWH and insomnia further research is needed in order to better understand factors relevant to HIV status that may have an impact on insomnia within PLWH population.

\section{CONCLUSION}

In conclusion, there are very limited number of studies examining insomnia treatment for PLWH. Of the treatments provided, those stemming from a cognitive behavioral treatment tended to have stronger positive outcomes in relation to one's sleep quality and quantity versus other alternative treatments. Future research should focus on the development of a CBT-I for PLWH with a focus on the particular determinants of insomnia in PLWH.

\section{REFERENCES}

American Psychiatric Association. (2013). Diagnostic and statistical manual of mental disorders: DSM-5: Author.

Arksey, H. and O'Malley, L. (2005). Scoping studies: towards a methodological framework. International Journal of Social Research Methodology, 8(1), 19-32. https://doi.org/ 10.1080/1364557032000119616.

Buchanan, D. T., McCurry, S. M., Eilers, K., Applin, S., Williams, E. T., \& Voss, J. G. (2018). Brief behavioral treatment for insomnia in persons living with HIV. Behavioral Sleep Medicine, 16(3), 244-258. https://doi.org/10.1080/15402002.2016.11 88392.

Bhaskar, S., Hemavathy, D., Prasad, S. (2016). Prevalence of chronic insomnia in adult patients and its correlation with medical conditions. Journal of Family Medicine Primary Care, 5(4), 780-784. https://doi.org/10.4103/2249-4863.201153.

Chung, KF., Lee, CT., Yeung, WF., Chan, MS., Chung, EWY. \& Lin, WL. (2018). Sleep hygiene education as a treatment of insomnia: a systematic review and meta-analysis. Family Practice, 35(4), 365-375. https://doi.org/10.1093/fampra/cm×122.

Dreher HM (2003). The effect of caffeine reduction on sleep quality and well-being in persons with HIV. Journal of Psychosomatic Research, 54(3),191-8. https://doi.org/10.1016/S00223999(02)00472-5. 
Epstein D., Sidani, S., Bootzin, R., Belyea, M. (2012). Dismantling multicomponent behavioral treatment for insomnia in older adults: a randomized control trial. Sleep, 35(6), 797 805. https://doi.org/10.5665/sleep.1878.

Garland, S., Rowe, H., Repa, L., Fowler, K., Rhou, E., Grandner, M. (2018). A decade's difference: 10 -year change in insomnia symptom prevalence in Canada depends on sociodemographics and health status. Sleep Health, 4(2), 160-165. https://doi.org/10.1016/j.sleh.2018.01.003.

Geiger-Brown, J., Rogers, V., Liu, W., Ludeman, E., Downton, K., Diaz-Abad, M. (2015). Cognitive behavioral therapy in persons with comorbid insomnia: a meta-analysis. Sleep Medicine Reviews, 23, 54-67. https://doi.org/10.1016/j.smrv.2014.11.007

Gong, H., Ni, CX., Liu, YZ., Zhang, WJ., Lian, YJ., Peng, W. \& Jiang, CL. (2016). Mindefulness meditation for insomnia: a meta-analysis of randomized controlled trials. Journal of Psychosomatic Research, 89, 1-6. https://doi.org/10.1016/j.jpsychores.2016.0 7.016.

Hudson, A. L., Portillo, C. J., \& Lee, K. A. (2008). Sleep disturbances in women with HIV or AIDS: Efficacy of a tailored sleep promotion intervention. Nursing Research, 57(5), 360366. https://doi.org/10.1097/01.NNR.000031350 1.84604.2c.

Jabbari, F., Dadaghzadeh, F., Khalili, H., Abbasian, L. (2015). Associated factors of sleep quality in HIV-positive individuals. Future virology, 10(2), 98-96. https://doi.org/10.2217/fvl.14.107

Jean-Louis G., Weber, K., Aouizerat, B., Levine, A., Maki, P., Liu, C., Anastos, K., Milam, J., Althoff, K., Wilson, T. (2012). Insomnia symptoms and HIV infection among participants in the womens interagency HIV study. Sleep, 35(1), 131-137. https://doi.org/10.5665/sleep.1602.

Johnson, J., Rash, J., Campbell, T., Savard, J., Gehrman, P., Perlis, M., Carlson, L., Garland, S. (2016). A systematic review and metaanalysis of randomized controlled trials of cognitive behavior therapy for insomnia (CBT-I) in cancer survivors. Sleep Medicine, 27, 20-28. https://doi.org/10.1016/j.smrv.2015.07.001

Low, Y., Goforth, H., Preud'homme, X., Edinger, J., Krystal, A. (2014). Insomnia in HIV-infected patients: pathophysiological implication. AIDS, 16(1), 3-13.

McDermott, A., Zaporojan, L., McNamara, P., Doherty, C. P., Redmond, J., Forde, C., ... Bergin, C. (2017). The effects of a 16-week aerobic exercise programme on cognitive function in people living with HIV. AIDS Care, 29(6), 667-674. https://doi.org/10.1080/09540121.2016.12 63723.

Miranda, J., Sidani, S., Fredericks, S., Fox, M. (2017). Sleep and cardiovascular effects of behavorial therapies for insomnia. British Journal of Cardiac Nursing, 12(10), 488-495. https://doi.org/10.12968/bjca.2017.12.10.4 88.

Medic, G., Wille, M., Hemels, M. (2017). Short and long-term health consequences of sleep disruption. Nature and Science of Sleep, 9, 151-161. https://doi.org/10.2147/NSS.S134864.

Morgenthaler, T., Kramer, M., Alessi, C., Friedman, L., Boehlecke, B., Brown, T., ... Swick, T. (2006). Practice parameters for the psychological and behavioral treatment of insomnia: An update. An American Academy of Sleep Medicine report. Sleep, 29(11),1415-1419.

Morin, C., Beaulieu-Bonneau, S., Cheung, J. (2019). Treatment of insomnia. In: Savard, J. \& Ouellet MC (Eds.), Handbook of sleep disorders (pp. 27-50). Elsevier. https://doi.org/10.1016/C22016-0-03652-5.

Morin, C.M. , Bootzin, R.R. , Buysse, D.J. , Edinger, J.D. , Espie , C.A. , \& Lichstein , K.L. (2006). Psychological and behavioral treatment of insomnia: Update of the recent evidence $(1998-2004)$. Sleep, 29(11),1398-1414. https://doi.org/10.1093/sleep/29.11.1398.

Morin, C., LeBlanc, M., Bélanger, L., Ivers, H., Mérette, C., Savard, J. (2011). Prevalence of insomnia and its treatment in Canada. The Canadian Journal of Psychiatry, 56(9), 540548. https://doi.org/10.1177/070674371105600 905. 
Ohayon, M. (2002). Epidemiology of insomnia: what we know and what we still need to learn. Sleep Medicine Review, 6(2), 97-111. https://doi.org/10.1053/smrv.2002.0186.

Phillips, K. D., \& Skelton, W. D. (2001). Effects of individualized acupuncture on sleep quality in HIV disease. JANAC: Journal of the Association of Nurses in AIDS Care, 12(1), 27-39. https://doi.org/10.1016/S10553290(06)60168-4.

Qaseem A, Kansagara D, Forciea MA, Cooke, M. Denberg, T. (2016) Management of chronic insomnia disorder in adults: a clinical practice guideline from the American College of Physicians. Annals Internal Medicine, 165,125-33. https://doi.org/10.7326/M15-2175.

Rogers, B., Lee, J., Bainter, S., Bedoya, A., Pinkston, M., Safren, S. (2018). A multilevel examination of sleep, depression, and quality of life in people living with HIV/AIDS. Journal of Health Psychology, Sep25(10-11), 1-11. https://doi.org/10.1177/135910531876563 2.

Sandoval R, Roddey T, Giordano TP, Mitchell K, Kelley C. (2016). Randomized trial of lower extremity splinting to manage neuropathic pain and sleep disturbances in people living with HIV/AIDS. Journal of the International Association of Providers of AIDS Care, 15(3), 240-7.

https://doi.org/10.1177/232595741351111 2.

Sidani, S., Epstein, D.R., Fox, M. (2019). Comparing the Effects of Single and Multiple Component Therapies for Insomnia on Sleep Outcomes. Worldviews on EvidenceBased Nursing, 16 (3), 195-203. https://doi.org/10.1111/wvn.12367.

Sim, J. (2019). Should treatment effects be estimated in pilot and feasibility studies? Pilot and Feasibility Studies, 5(107). https://doi.org/10.1186/s40814-019-04937.

Straten, A., Zweerde, T., Kleiboer, A., Cuijpers, P., Morin, C., Lancee, J. (2017). Cognitive and behavioral therapies in the treatment of insomnia: a meta-analysis. Sleep Medicine Reviews, 38, 3-16. https://doi.org/10.1016/j.smrv.2017.02.001
Taibi, D. (2013). Sleep disturbances in persons living with HIV. Journal of the Association of Nurses in AIDS Care, 24(1), S72-S85. https://doi.org/10.1016/j.jana.2012.10.006.

Taylor, D. \& Pruiksma, K. (2014). Cognitive and behavioural therapy for insomnia (CBT-I) in psychiatric populations: A systematic review. International Review of Psychiatry, 26(2), 205-213. https://doi.org/10.3109/09540261.2014.90 2808.

Trauer, J., Qian, M., Doyle, J., Rajaratnam, S., Cunnignton, D. (2015). Cognitive behavioral therapy for chronic insomnia: a systematic review and meta-analysis. Annals of Internal Medicine, 163(3), 191-204. https://doi.org/10.7326/M14-2841.

Troxel, W.M., Germain A. \& Buysse, D.J. (2012). Clinical management of insomnia with brief behavioral treatment (BBTI). Behavioral Sleep Medicine, 10(4), 266-279. https://doi.org/10.1080/15402002.2011.60 7200.

Van Stratten, A., van der Zweerde, T., Kleiboer, A., Cuijpers, P., Morin, C. \& Lancee, J. (2018). Cognitive and behavioral therapies in the treatment of insomnia: A meta-analysis. Sleep Medicine Reviews, 38, 3-16. https://doi.org/10.1016/j.smrv.2017.02.001

Webel, A., Moore, S., Hanson, J., Patel, S., Schmotzer, B., Salata, R. (2013). Improving sleep hygiene behavior in adults living with HIV/AIDS: a randomized control pilot study of the systemchange HIV intervention. Applied Nursing Research, 26(2), 85-91. https://doi.org/10.1016/j.apnr.2012.10.002

Wu, J., Appleman, E., Salazar, R., Ong, J. (2015). Cognitive behavioral therapy for insomnia comorbid with psychiatric and medical conditions a meta-analysis. Journal of the American Medical Association Internal Medicine, 175(9), 1461-1472. https://doi.org/10.1001/jamainternmed.20 15.3006.

Wu, J., Lu, C., Guo, L., Li, P. (2015). Self-reported sleep disturbances in HIV-infected people: a meta-analysis of prevalence and moderators. Sleep Medicine, 16(8), 901907. 
International Health Trends and Perspectives

https://doi.org/10.1016/j.sleep.2015.03.02

7.

Zielinski, McKenna \& McCarley (2016). Functions and mechanisms of sleep, AIMS Neuroscience, 3(1), 67-104.

https://doi.org/10.3934/Neuroscience.2016 .1.67. 
Figure 1: Article Flow Diagram

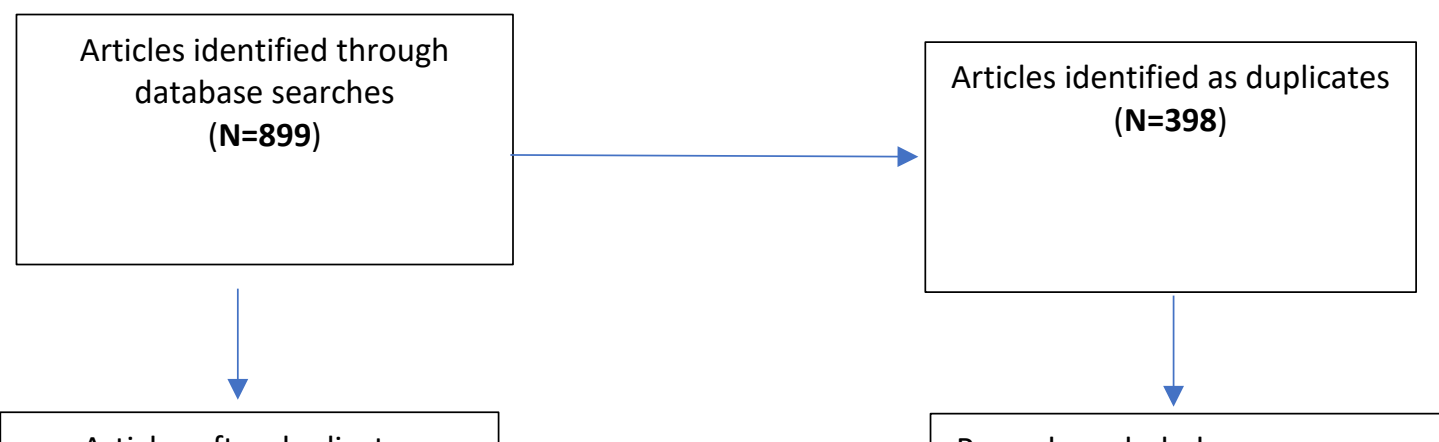

Articles after duplicates removed, title and abstract reviewed for eligibility

( $N=501)$

$(\mathrm{N}=494)$

Rationale for exclusion:

1. Population was PLWH but no focus on insomnia ( $\mathbf{N}=\mathbf{2 7 9}$ )

2. Articles described insomnia in PLWH but did not involve treatment $(\mathrm{N}=126)$

Full-text articles reviewed for eligibility

$(\mathbf{N}=7)$

3. Pharmacological treatment addressed for insomnia in PLWH ( $\mathbf{N = 5})$

4. Included insomnia but with no focus on PLWH ( $\mathrm{N}=\mathbf{2 2}$ )

5. Main focus was not on insomnia, PLWH or treatment $(\mathbf{N}=\mathbf{6 2}$ )

Studies included in the scoping review $(\mathrm{N}=7)$ 
Table 1: Summary of study characteristics used in the scoping review

\begin{tabular}{|c|c|c|c|c|c|c|c|}
\hline SOURCE & YEAR & COUNTRY & $\begin{array}{l}\text { NUMBER } \\
\text { OF } \\
\text { SUBJECTS }\end{array}$ & DESIGN & $\begin{array}{l}\text { SLEEP } \\
\text { MEASURES }\end{array}$ & TREATMENT TYPES & $\begin{array}{l}\text { COMPARISON } \\
\text { TREATMENT }\end{array}$ \\
\hline $\begin{array}{l}\text { Buchannan et } \\
\text { al. [26] }\end{array}$ & 2018 & U.S.A & $\mathrm{N}=12$ & $\begin{array}{l}\text { One group quasi- } \\
\text { experimental } \\
\text { with } \\
\text { pretest/posttest } \\
\text { measures }\end{array}$ & $\begin{array}{l}\text { PSQI } \\
\text { Sleep Diary } \\
\text { PROMIS } \\
\text { ISI }\end{array}$ & $\begin{array}{l}\text { Brief Behavioral } \\
\text { Treatment for Insomnia: } 4 \\
\text { weekly sessions: two in } \\
\text { person (sessions 1,3) and } \\
\text { two by telephone } \\
\text { (sessions } 2,4 \text { ). } \\
\text { Focus is on behavioral } \\
\text { components of insomnia } \\
\text { treatment. }\end{array}$ & $\mathrm{N} / \mathrm{A}$ \\
\hline Dreher [29] & 2003 & $\begin{array}{l}\text { U.S.A, } \\
\text { Canada, } \\
\text { Brasil }\end{array}$ & $\mathrm{N}=88$ & $\begin{array}{l}\text { Two-group } \\
\text { randomized } \\
\text { control study }\end{array}$ & PSQI & $\begin{array}{l}\text { Instructed to withdraw } \\
\text { from caffeine using a } \\
\text { Gradual withdraw from } \\
\text { caffeine protocol and then } \\
\text { asked to avoid all caffeine } \\
\text { sources for } 30 \text { days. }\end{array}$ & $\begin{array}{l}\text { Instructed to } \\
\text { maintain their } \\
\text { ordinary and usual } \\
\text { daily caffeine } \\
\text { consumption } \\
\text { without variation. }\end{array}$ \\
\hline $\begin{array}{l}\text { Hudson et al. } \\
\text { [27] }\end{array}$ & 2008 & U.S.A & $\mathrm{N}=30$ & $\begin{array}{l}\text { Pretest/posttest } \\
\text { pre-experimental } \\
\text { design }\end{array}$ & $\begin{array}{l}\text { PSQI } \\
\text { Wrist } \\
\text { actigraphy } \\
\text { Sleep diary } \\
\text { GSDS }\end{array}$ & $\begin{array}{l}\text { Educational and } \\
\text { behavioral set of sleep- } \\
\text { promoting behaviors } \\
\text { based on principles of } \\
\text { sleep hygiene. } \\
\text { A 30-40min individual } \\
\text { session in which six } \\
\text { primary principles of sleep } \\
\text { hygiene were reviewed. } \\
\text { Participants contracted to } \\
\text { try two of the six sleep } \\
\text { hygiene behaviors for 1- } \\
\text { week. }\end{array}$ & $\mathrm{N} / \mathrm{A}$ \\
\hline
\end{tabular}


International Health Trends and Perspectives

\begin{tabular}{|c|c|c|c|c|c|c|c|}
\hline $\begin{array}{l}\text { McDermott et } \\
\text { al. [31] }\end{array}$ & 2017 & Ireland & $\mathrm{N}=11$ & $\begin{array}{l}\text { Two-group } \\
\text { randomized } \\
\text { control study }\end{array}$ & PSQI & $\begin{array}{l}\text { 16-week aerobic exercise } \\
\text { program }\end{array}$ & $\begin{array}{l}\text { No intervention } \\
\text { received. } \\
\text { Participants were } \\
\text { advised to continue } \\
\text { their normal daily } \\
\text { routine. }\end{array}$ \\
\hline $\begin{array}{l}\text { Phillips and } \\
\text { Skelton [28] }\end{array}$ & 2001 & U.S.A & $N=21$ & $\begin{array}{l}\text { Pretest/posttest } \\
\text { pre-experimental } \\
\text { design }\end{array}$ & $\begin{array}{l}\text { PSQI } \\
\text { Wrist } \\
\text { actigraph } \\
\text { CSQI }\end{array}$ & $\begin{array}{l}\text { Acupuncture treatment } \\
\text { provided } 2 \text { evenings per } \\
\text { week for } 5 \text { weeks for a } \\
\text { total of } 10 \text { sessions. Each } \\
\text { session lasting } 30 \text { to } 45 \\
\text { mins. }\end{array}$ & N/A \\
\hline $\begin{array}{l}\text { Sandoval et al. } \\
{[30]}\end{array}$ & 2016 & U.S.A & $N=46$ & $\begin{array}{l}\text { Two-group } \\
\text { randomized } \\
\text { control trial }\end{array}$ & PSQI & $\begin{array}{l}\text { Night time wearing of } \\
\text { bilateral lower extremity } \\
\text { splints for } 6 \text {-weeks }\end{array}$ & $\begin{array}{l}\text { Use of parallel } \\
\text { splint liner for 6- } \\
\text { weeks }\end{array}$ \\
\hline $\begin{array}{l}\text { Webel et al. } \\
{[22]}\end{array}$ & 2013 & U.S.A & $N=40$ & $\begin{array}{l}\text { Two-group } \\
\text { randomized } \\
\text { control study }\end{array}$ & $\begin{array}{l}\text { Wrist } \\
\text { actigraph } \\
\text { PROMIS }\end{array}$ & $\begin{array}{l}\text { SystemCHANGE-HIV } \\
\text { Intervention that included } \\
\text { 10-weekly group sessions } \\
\text { on different topics of HIV } \\
\text { management. }\end{array}$ & $\begin{array}{l}\text { Control group } \\
\text { received a copy of } \\
\text { the HIV Symptom } \\
\text { Management } \\
\text { Strategies: A } \\
\text { Manual for People } \\
\text { Living with } \\
\text { HIV/AIDS }\end{array}$ \\
\hline
\end{tabular}

